\title{
Modeling and Developing a System of Information Systems for Managing Heterogeneous Resources
}

\author{
Majd Saleh, Marie-Hélène Abel \\ Sorbonne Universités, Université de Technologie de Compiègne \\ CNRS, HEUDIASYC, UMR 7253, CS 60319 \\ 60203 Compiègne cedex - France \\ Email: \{majd.saleh,marie-helene.abel $\} @$ utc.fr
}

\begin{abstract}
Organizations struggle to manage overwhelming amount of heterogeneous resources, which are produced by several users working on different projects using various Information Systems. This paper takes the task for modeling and developing a System of Information Systems (SoIS) for managing heterogeneous resource. In order to manage these resources we are following the approach of SoIS that can be viewed as a single entry point for several Information Systems granting the user access to information and resources produced from multiple autonomous Information Systems. It can provide the ability to capitalize the knowledge of users as they are interacting with Information Systems and each other. In this context, we look into the ability to index, share, and annotate important resources in the environment of the SoIS. In that sense, this paper focuses on modeling and developing a System of Information Systems (SoIS) for managing heterogeneous resource.
\end{abstract}

\section{INTRODUCTION}

\section{A. Social Context}

In recent years there has been unprecedented growth in organizations reliance on partnering and various forms of collaboration. Today, nearly most of the viral tasks taking place in organizations are carried out by different users working on various Information Systems [1]. This is made possible as a consequence of the recent growth of communication technologies and Information Systems. As consequence, organizations depend on many teams to carry out the necessary tasks and achieve their goals. Those teams depend on many Information Systems to execute the delegated tasks. In the process of working at this manner, numerous amounts of digital heterogeneous resources are produced. In overall view, organizations are left alone to deal with the resulted overwhelming amount of heterogeneous resources distributed over different standalone and/or web-based applications.

As a general rule, time and effort are always in a direct proportion. The best case scenario is to break the ratio and reduce time without increasing effort. The "time" we seek to reduce is the time required to find, review, discuss, evaluate and access various resources produced from different Information Systems.

\section{B. Scientific Context}

System of Systems (SoS) are collections of dedicated systems that combine their resources and capabilities to create a new, more complex system that offers more functionality than simply the sum of its member systems [2] . In our context, we are particularly interested in a category of SoS: Systems of Information Systems (SoIS). A SoIS can be considered as a macro-information system giving access to information distributed in component systems and offering functionality using the accessed information. In order to facilitate the organization of resources within an organization, we have chosen to follow the approach of System of Information Systems (SoIS) and to develop a support platform based on a collaborative model.

Within the environment of the SoIS, overwhelming amount of heterogeneous resources is shared by collaborators on different Information Systems. These resources, if managed properly, could improve the understanding of collaborators in their field of expertise [3]. This improvement can be achieved through establishing a common ground for collaborators to work together with the overwhelming amount of the heterogeneous resources. The main problem addressed by this paper is how to find the common ground for collaborator to manage heterogeneous resources in the SoIS? In more details, how to index, share and annotate resources in the SoIS?

\section{Plan}

This paper is organized as follows: section 2 presents related work and positions our work with its context. In section 3 , the model and the prototype of the SoIS are presented. In section 4, a discussion of MEMORAeSoIS prototype takes place. Finally, this paper is concluded with the prospective ideas and features of the SoIS in section 5.

\section{RELATED WORK}

This section looks into related researches focused on collaboration and resources management, and positions our work in that context.

The work in [4] is about collaborative software development platforms for crowdsourcing. According to [4], in crowd- 
sourced software development, enterprises delegate many software engineering tasks to external individuals or groups with the support of crowdsourcing platforms. Large IT companies use internal crowdsourcing with software development tasks and their own employees to leverage untapped human resources. The crowdsourcing platform supports the exchange of messages and information among requesters and providers to reduce gaps and ambiguity. It also supports various collaborations by providing the facilities for sharing workspaces and encouraging user interactions. We argue that such platforms though possess great potentials in terms of communication and collaboration; they lack the ability to open discussion about the work deliverable. Also, it is not possible to reference any resources in those platforms.

In [5], authors emphasize on the benefits of a Shared Workspace System in a group collaboration platform that allows the creation of documents, appointments, contacts, tasks and notes within shared workspaces. Using only a web browser, without having to install additional software, the users can access the data anytime, from anywhere in the world. Yet again, the mechanism for organizing the resources produced in such system is missing. To our understanding, sharing spaces are the corner stones of any collaborative platform, but the missing ingredient here is the key to index the resources for quick access and retrieval.

Another collaboration platform discussed in [5] and [6] is phpGroupWare platform. phpGroupWare is a fully featured, web based messaging, collaboration and enterprise management platform. It is provided with a range of modules (more than 50 applications) that can be selected and installed according to needs. Some of the most powerful features that can be used for multinational team collaboration are: contacts management, email, shared web-based calendar, to-do lists, address book, web content and document management and sharing. One issue we can mark about this product is that most of the organizations already have specialized software for communication, document creation, contact management, etc. What we should focus on in a collaboration platform is the ability to incorporate existent solutions and index their resources.

The aforementioned examples of collaboration platform indicate the need for an encompassing solution to the problem of resources management that takes into consideration the organizations need to communication and sharing, while preserving the autonomous nature of the existing Information systems.

The notion of System of Information Systems is defined by [7] as "networks of agents interacting in a specific technology area under a particular institutional infrastructure for the purpose of creating, diffusing, and utilizing technology focused on knowledge, information, and competence flow." [8] describes SoIS as "the specific clusters of the firms, technologies, and industries involved in the generation and diffusion of new technologies and in the knowledge flow that takes place among them. As mentioned in [9], there are different approaches concerning the coordination of Systems in a distributed environment such as SoIS. We choose primarily to focus on the approach of Leader/Follower. In the Leader/Follower approach, a System is designated as the leader of the orchestration of the SoIS while the other systems are designated as followers that can link to the SoIS by following the leader. According to [10], the leader system should provide an added value to the digital ecosystem in which it is operating, by allowing for more communication among the different systems participating in the SoIS.

\section{OUR PROPOSAL}

Within the context of our research, the leader system should emphasize on these points:

- The importance to share resources with a community in order to achieve a common goal.

- The fact that shared resources can come from different information systems.

- The references for shared resources should be stored and retrieved easily.

Based on these points we can view the leader system as the organizer of the SoIS. It can be considered to contain a knowledge base that holds all the necessary information of the SoIS (users' accounts, activities log, references of shared resources, etc.). It should also control the communication line linking all the composing Information Systems to the SoIS. In addition, it should allow the sharing, indexing, and accessing the references resources in the SoIS. Ultimately, the leader system is responsible for providing all the services for the users in the SoIS to facilitate collaboration and decision making process.

This section will start by giving a use case to illustrate the benefits of having an SoIS. Then, the choice of MEMORAEcore 2 model is discussed and the model of the SoIS is provided. After that, the prototype of the SoIS is shown.

\section{A. Use Case}

For the sake of explaining our proposal, we take the following scenario in the discourse of a computer science course at the University of Technology of Compiegne: Several groups of students are enrolled in a programming course. Students use many standalone and web-based applications during this course. Students use web links for tutorials and useful information, University portal that contains resources about the course which can be also considered as a web link, wiki pages to document certain subjects, note taking applications, contact lists to save important contacts of their instructors and colleagues, a system to upload and share their snippets of code with each other's and their instructors, and even social media networks sometimes to discuss about the material of the course or follow certain announcements. It is 


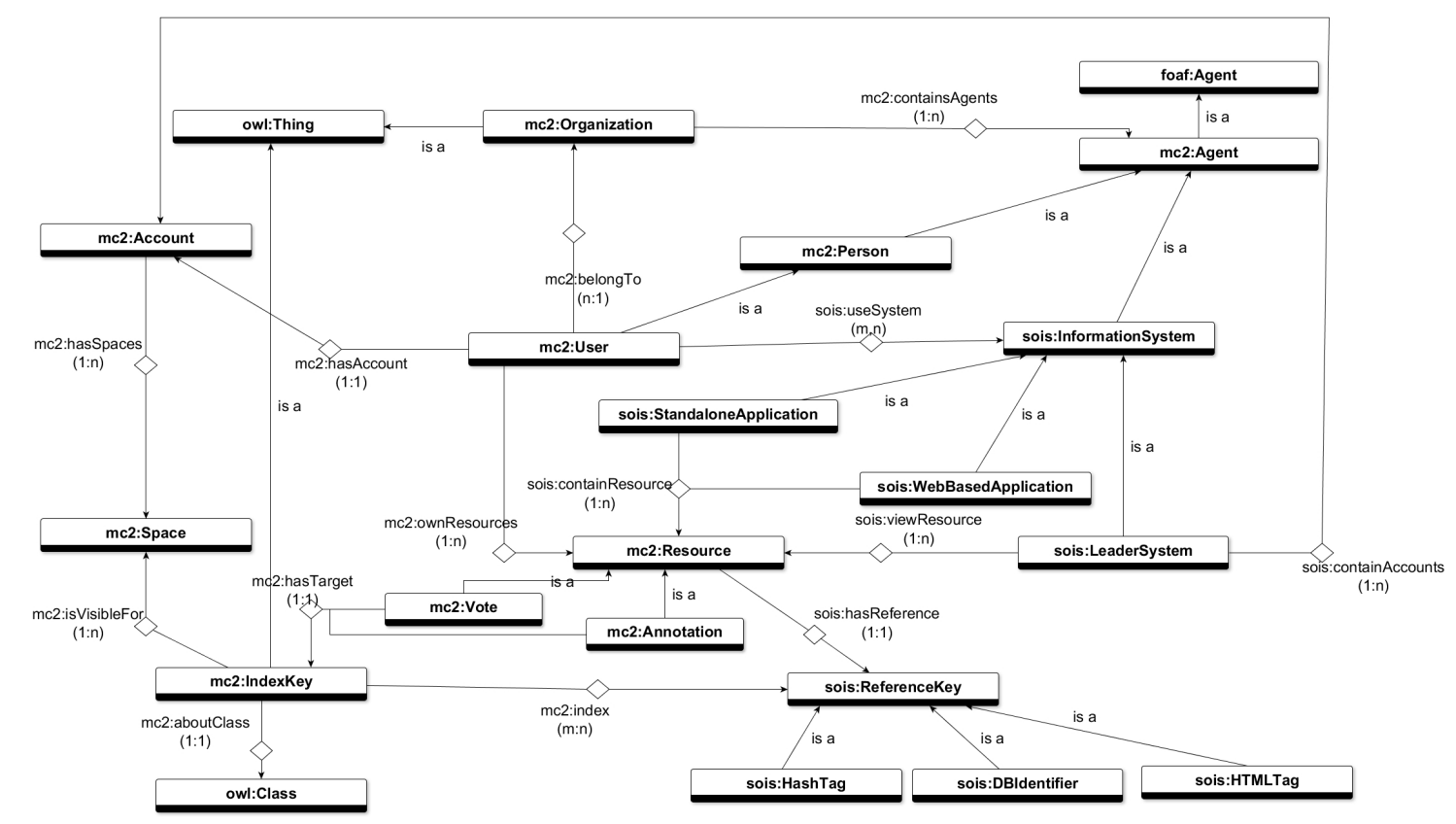

Fig. 1. Semantic model of the SoIS.

certain that different resources coming from different system are related to the same topic. It is considerably more efficient for students to be able to find all the resources together under the same topic. This eventually will extrapolate the problem of wasted time trying to find the right resources for the right user. It will ultimately lead into having an indexing schema where only important resources are indexed together based on shared hierarchy of terminology. Using such indexing schema will allow having a heterogeneous mix of resources indexed together under a certain concept and work to complement each other. Furthermore, this indexing will allow for further functionalities like being able to comment on resources and vote on them to enrich the information flow in the company. In addition to that, the comments themselves can be considered as resources and have their own index. A comment about a resource indexed under a certain concept of a certain course, can apply to another concept of another course and could be indexed there as well. This method of dealing with comments as resources on their own has proved to be very effective in the discourse of organizational learning according to [11].

\section{B. The Choice of MEMORAe Model}

In order to develop the model of the SoIS and build its first prototype, we start by using a model for knowledge management called MEMORAe-core2. The MEMORAe-core2 model employs the Semantic Web standards [12] (SIOC, FOAF, BIBO). The model of MEMORAe-core2 considers an organization as a group of members interacting with each other's. Those members are modeled as users with the MEMORAe-core2 model. Every user and group of users is associated with one Sharing space that will allow the sharing of heterogeneous resources. The model also provides the user with access to several knowledge bases in the form of a semantic map of concepts. The concepts of the map represent an indexing schema for users to index their resources. In addition, MEMORAe-core2 model gives the users the ability to annotate resources to highlight certain ideas. The annotations themselves are treated as resources, and can be indexed and shared separately.

Developed from the MEMORAe-core2 model, the SoIS has purpose of offering users the following features:

- Accessing resources coming from different information systems in one Centralized location.

- Creating resources using a dedicated information system. These resources can be accessible either from the SoIS or from the dedicated system where they are created.

- Organizing resources around a shared repository presented in the form of a map.

- Sharing resources in different sharing spaces,

- Annotating resources to highlight some related ideas.

\section{Model of MEMORAeSoIS}

In this section we move forward from MEMORAe-core2 model towards a model for SoIS. It is useful to take into account the standard representation of resources in MEMORAecore 2 model and build upon it the representation of resources 


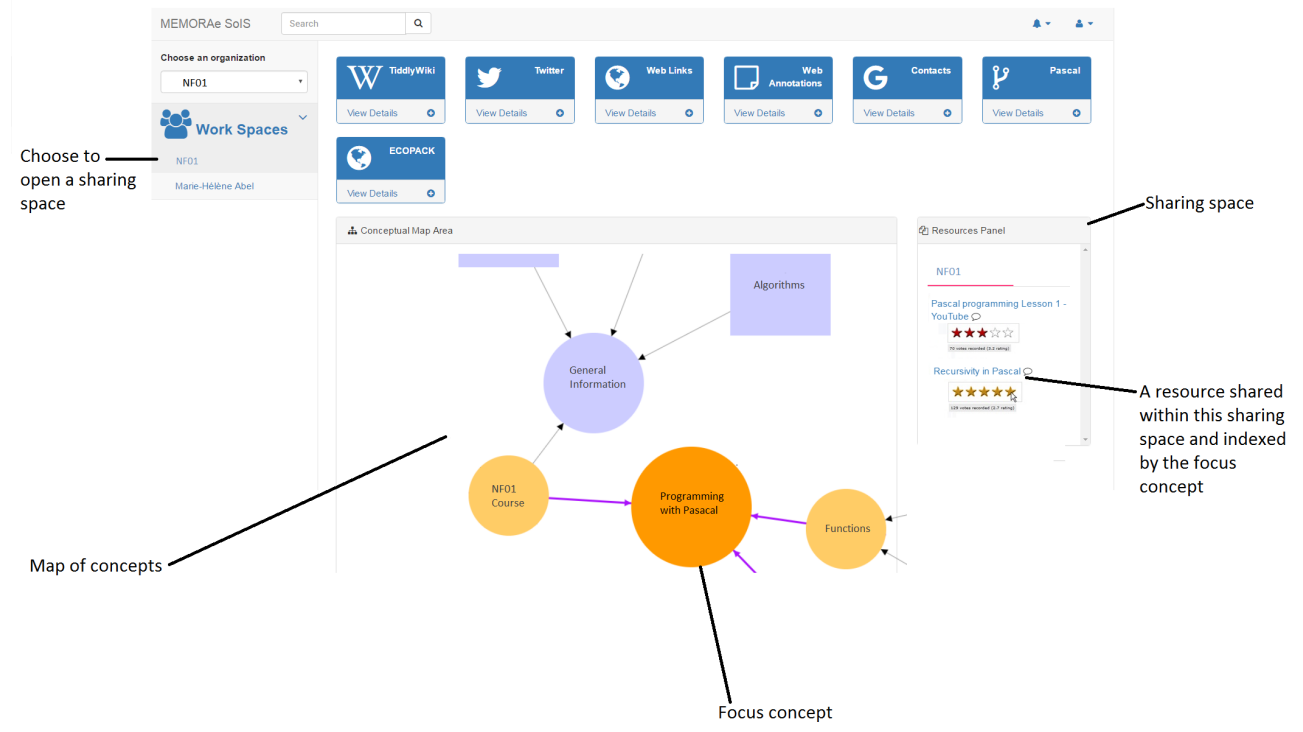

Fig. 2. SoIS Prototype main screen

used in SoIS. The model is presented in (Fig. 1). The model highlights the position of an agent, either as an Information System or as a user, in the orchestration of the SoIS. The model also shows how resources are created by users and contained in the composing systems of the SoIS, while the leader system is only showing those resources through a reference key. Each resource has a reference which functions as a link between the resource and the leader system. This key can be an HTML tag, a Database Identifier, or social bookmark as a Hash tag. Following the MEMORAe-core 2 model, the leader can view a semantic map of shared terminology in the work environment. The semantic map allows the indexing of references to the actual resources through the index key and sharing it within different sharing spaces.

We also deployed the function of voting so that users evaluate a resource by relevance of which it is indexed, and sharing space where the resource is visible. As shown in (Fig. 1), vote is a resource in the model of the SoIS. It is related with IndexKey by the relation hasTarget as shown in (Fig. 1).

The SoIS allows the users to interact with various heterogeneous resources coming from different Information Systems in many ways. The user can view the referenced resources, index the important ones, share them within different sharing spaces, annotate, comment and vote on them. An added value can be achieved by tracing the users' activities in the SoIS. The importance of these traces comes from their role in the reasoning process of the recommender system proposed by [13].

In the model of SoIS we can view the leader system as a knowledge base that handles storing, organizing, and sharing of resources from different Information Systems. MEMORAecore 2 model aims, by its design, to facilitate knowledge sharing and capitalization within those different systems. All types of resources can indexed by a map of shared terminology that represents the concepts of interest for the user [11]. Furthermore, the map defines a common reference shared between all users. The focus concept of the map is a concept in which the user is interested in. The shared resources are indexed by the concepts of the map. In this orchestration of SoIS, MEMORAeSoIS is introduced to new resources produced by Information Systems that reside outside its scope, hence the use of the leader system as it supports different types of social resources (wiki, forums, chat) and documentary resources (documents, images, web link). This constitutes an added value for the Information Systems comprising the SoIS, as data produced from those systems is capitalized as knowledge within the leader.

\section{Prototype of MEMORAeSoIS}

The systems composing the SoIS are autonomous and work separately of each other. Each of which has its own services/functions and databases. While some systems are openly providing an API for requesting their services, especially the case with web-based applications, other systems are closed and operate as a black box. Information can be represented in different ways within different systems, thus, the SoIS might face some difficulties accessing resources, unless the services of that system are available through an API. More detailed illustration about the SoIS architecture can be found in [14].The added value of the SoIS is found in the ability to index resources using a map of shared terminology among col- 


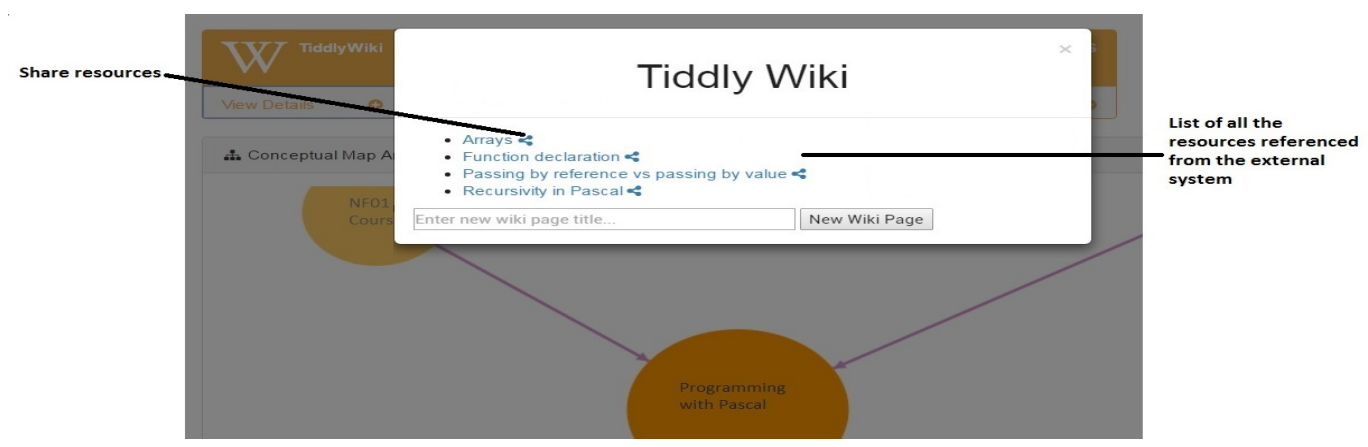

Fig. 3. Panel to access resources available in the TiddlyWiki, or create new ones.

laborators and share resources within different sharing spaces. That will give the users of the SoIS the ability to collaborate over resources and rate them. These features are not available when the Information Systems were working separately. In the premise of the SoIS the users can share resources from different external systems and vote on them with simplicity and ease.

Other than the leader system the prototype links various standalone and web-based applications that are different in their purpose and goals, hence they produce different types of resources. These systems are: TiddlyWiki (Web-Based), Twitter (Web-Based), Web-links (Web-Based), Web Annotations (Web-Based), Google Contacts (Web-Based), Pascal (Standalone), and ECOPACK Platform (Standalone).

The purpose of the prototype is to provide hands-on experience for our solution to the problem of managing heterogeneous resources in organizations through indexing, sharing, voting for, and annotating these resources with the help of a map of shared terminology. After login, the user can choose an organization that he/she works in. The user might have one or more organizations to choose from. Based on the chosen organization, the SoIS will view a map of concepts specific to that organization and can be used to index the heterogeneous resources. The user will also have access to the sharing spaces. For each user there is at least two sharing spaces; one global for the whole organization, and the other is the personal sharing space. Resources of the external systems are referenced in their respected tabs. The user can view all the referenced resources for each system and index the important ones based on the map of concepts, and share the indexed resources in a sharing space. Moreover, on each box that is used to access an external system there is a button with a "plus" sign. This button will allow the user to navigate through all the resources available in the dedicated Information System and select from the list of resources which are going to be indexed by the map of concepts and shared in certain sharing spaces. It also allows the user to create new resources in their respected Information System. The panel to navigate through resources or create new ones is shown in (Fig. 3). The resources of the dedicated Information System is made available to the user by means of the data wrapper and the server/observer endpoint

After indexing the important resources, users are able to annotate and vote for those resources. The annotation and votes are themselves considered as resources. They, as well, can be indexed and shared in their own right.

For the prototype to be tested we currently collect feedback data from students at the University of Technology of Compiegne (UTC) to evaluate the preference of the SoIS. The students are enrolled in a computer science course focused on the subjects of algorithms and programming. We will use their feedback on the SoIS based on two aspects; a survey form, and hands-on sessions with the SoIS. For the setup of this test, each student is provided with an account in the SoIS. This account has access to a map of concepts representing the ontology of the course in which students are enrolled. Students then can index, share, annotate, and vote on heterogeneous resources related to this course. Also, each student has three sharing spaces in the platform: a personal sharing space, a sharing space for the group in which the student belongs, and a last sharing space with the instructor of the course.

\section{Discussion}

In this section we will elaborate on the different fields of application of MEMORAeSoIS.

The core functionalities of MEMoRAeSoIS are indexing, sharing, annotating, accessing resources, and voting for resources. These functionalities apply to all kind of heterogeneous resources managed by MEMoRAeSoIS. However, the resources themselves are different as they come from different Information Systems. In that sense, the domain of application for MEMoRAeSoIS depends on the type of resources presented within its member systems. For example, if we are in the domain of e-learning, the Information systems and their resources should revolve around this domain.

Regarding the information flow between the leader system and the other member systems, information marches from the member Information System to the leader system as references of resources only. The leader system does not have a global view on the information of its member systems. 
For the moment, it only needs a reference of the resources residing in the member system to index it based on a map of shared terminology. It would be a good practice to discuss the possibility having a global view of the member systems within the leader system of the SoIS. This, however, will increase liability over the observer server responsible for this kind of communication.

The work in [15] presents a Communicating Structures Library (CSL) for System of Systems (SoS). The Communicating Structure is a hierarchical and concurrent structure that represents the SoS components and communication between them. The system components are represented simply as nodes. The nodes have memory that may contain items. Nets are sets of links that connect the nodes. The items are generated in some nodes and move from node to node along links concurrently and with some delay. Although this study shows promising results regarding performance enhancement in a complex structure like SoS, from our point of view, it fails to address the autonomous nature of the SoS. The data can flow from node to node, which means from system to system. In that case the member systems of SoS lose their autonomous nature as they depend on external data to function. Nevertheless, this Communication Structure can be utilized in our collaborative SoIS to remodel the communication medium in our architecture for better performance.

\section{CONClusion AND Future Work}

The goal of this paper was modeling and developing a System of Information Systems for managing heterogeneous resources. The heterogeneous resources are the result of different users working on different projects, using several Information Systems in the digital environment of organizations. The aim was focused towards investigating the leader system role in facilitating resources management in a System of Information Systems (SoIS), and to model and develop MEORAeSoIS. To achieve this goal this paper undertakes an effort to present the social and scientific context of this research and define the state of the art, then move to present a use case scenario that helped in realizing the model of the SoIS composed of various Information Systems with the inspiration of MEMORAe-core2 model. This paper found potentials in adapting the MEMORAe-core2 to manage the resources produced by different systems in the SoIS. It was also clear that combining resources from various Information Systems and manage them within the leader system will result in an added value to users not present when those systems were operating separately. The added value that we aim to achieve in MEMORAeSoIS prototype is the ability to trace users' activities. Furthermore, the SoIS can upgrade this value by providing analysis of these activities to determine users' competence levels at certain subjects or importance level of concepts.

The next step is to expand our work to implement the users' trace and introduce new Information Systems to MEMORAeSoIS based on the prototype presented in this paper and users' needs. MEMORAeSoIS should keep simple interface, with all the resources as far from the user as a single click, to keep the users experience useful and friendly.

\section{ACKNOWLEDGMENT}

This project is done under ECOPACK project and funded by ANR-ASTRID programme.

\section{REFERENCES}

[1] R. S. Burt, Structural holes: The social structure of competition. Harvard university press, 2009.

[2] S. W. Popper, S. C. Bankes, R. Callaway, and D. DeLaurentis, "System of systems symposium: Report on a summer conversation," Potomac Institute for Policy Studies, Arlington, VA, vol. 320, 2004.

[3] E. Deparis, M.-H. Abel, G. Lortal, and J. Mattioli, "Information management from social and documentary sources in organizations," Computers in Human Behavior, vol. 30, pp. 753-759, 2014.

[4] X. Peng, M. A. Babar, and C. Ebert, "Collaborative software development platforms for crowdsourcing." IEEE software, vol. 31, no. 2, pp. 30-36, 2014.

[5] M. Bîzoi, A. Suduc, and F. Filip, "Using collaborative platforms for decision support," in Proceedings of the 17th International Conference on Control Systems and Computer Science. Citeseer, 2009, pp. 349 352.

[6] M. Bîzoi, A.-M. Suduc, G. Gorghiu, L.-M. Gorghiu, I. Rudas, M. Demirafp, and N. Mastorakis, "Rates on collaborative platforms activity in multinational educational projects," in WSEAS International Conference. Proceedings. Mathematics and Computers in Science and Engineering, no. 9. WSEAS, 2009.

[7] B. Carlsson and R. Stankiewicz, "On the nature, function and composition of technological systems," Journal of evolutionary economics, vol. 1, no. 2, pp. 93-118, 1991.

[8] S. Breschi and F. Malerba, Sectoral innovation systems: technological regimes, Schumpeterian dynamics and spatial boundaries. Centro studi sui processi di internazionalizzazione, Università commerciale'Luigi Bocconi', 1996.

[9] R. Lozano, M. W. Spong, J. A. Guerrero, and N. Chopra, "Controllability and observability of leader-based multi-agent systems," in Decision and Control, 2008. CDC 2008. 47th IEEE Conference on. IEEE, 2008, pp. 3713-3718.

[10] H. Dong and F. K. Hussain, "Digital ecosystem ontology," in Industrial Electronics, 2007. ISIE 2007. IEEE International Symposium on. IEEE, 2007, pp. 2944-2947.

[11] A. Atrash, M.-H. Abel, C. Moulin, N. Darène, F. Huet, and S. Bruaux, "Note-taking as a main feature in a social networking platform for small and medium sized enterprises," Computers in Human Behavior, vol. 51, pp. 705-714, 2015 .

[12] A. Atrash, M.-H. Abel, and C. Moulin, "Supporting organizational learning with collaborative annotation," in International Conference on Knowledge Management and Information Sharing, 2014, pp. 237-244.

[13] N. Wang, M.-H. Abel, J.-P. Barthes, and E. Negre, "Mining user competency from semantic trace," in Computer Supported Cooperative Work in Design (CSCWD), 2015 IEEE 19th International Conference on. IEEE, 2015, pp. 48-53.

[14] M. Saleh, M.-H. Abel, V. Misséri, C. Moulin, and D. Versailles, "Integration of brainstorming platform in a system of information systems," in Proceedings of the 8th International Conference on Management of Digital EcoSystems. ACM, 2016, pp. 166-173.

[15] V. Kotov, Systems of systems as communicating structures. Hewlett Packard Laboratories, 1997. 